\title{
Isotope Dependence and Quantum Effects on Atomic Hydrogen Diffusion in Liquid Water
}

\author{
J. A. Walker, ${ }^{1}$ S. P. Mezyk, ${ }^{3}$ E. Roduner, ${ }^{4,5}$ and D. M. Bartels*,1,2 \\ ${ }^{1}$ Radiation Laboratory and Department of Chemistry \& Biochemistry, Notre Dame University, Notre Dame, Indiana 46556, United \\ States \\ ${ }^{2}$ Chemistry Division, Argonne National Laboratory, 9700 South Cass Avenue, Argonne, Illinois 60439, United States
}

${ }^{3}$ Department of Chemistry \& Biochemistry, California State University, 1250 Bellflower Boulevard, Long Beach, California 90840, United States

${ }^{4}$ Institute of Physical Chemistry, University of Stuttgart, Pfaffenwaldring 55, D-70569 Stuttgart, Germany

${ }^{5}$ Department of Chemistry, University of Pretoria, Pretoria 0002, South Africa

\begin{abstract}
Relative diffusion coefficients were determined in water for the $\mathrm{D}, \mathrm{H}$, and $\mathrm{Mu}$ isotopes of atomic hydrogen by measuring their diffusion-limited spin-exchange rate constants with $\mathrm{Ni}^{2+}$ as a function of temperature. $\mathrm{H}$ and $\mathrm{D}$ atoms were generated by pulse radiolysis of water and measured by time-resolved pulsed EPR. $\mathrm{Mu}$ atoms are detected by muonium spin resonance. To isolate the atomic mass effect from solvent isotope effect, we measured all three spin-exchange rates in $90 \% \mathrm{D}_{2} \mathrm{O}$. The diffusion depends on the atomic mass, demonstrating breakdown of Stokes-Einstein behavior. The diffusion can be understood using a combination of water "cavity diffusion" and "hopping" mechanisms, as has been proposed in the literature. The H/D isotope effect agrees with previous modeling using ring polymer molecular dynamics. The "quantum swelling" effect on muonium due to its larger de Broglie wavelength does not seem to slow its "hopping" diffusion as much as predicted in previous work. Quantum effects of both the atom mass and the water librations have been modeled using RPMD and a qTIP4P/f quantized flexible water model. These results suggest that the muonium diffusion is very sensitive to the $\mathrm{Mu}$ versus water potential used.
\end{abstract}

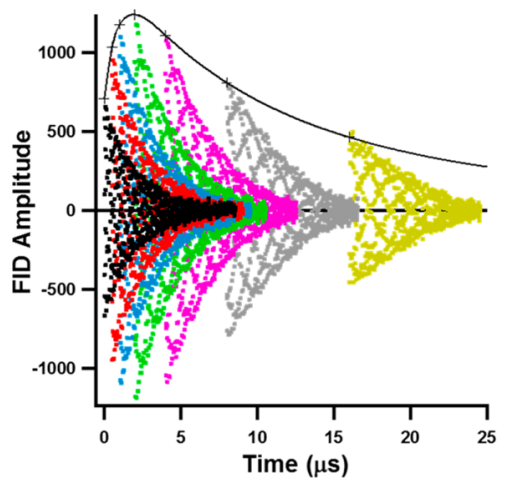

\section{Introduction}

It is common knowledge that proton diffusion in liquid water is very fast because it can take advantage of a special mechanism, the Grotthuss effect. ${ }^{1,2}$ Less well known is the fact that the diffusion coefficient of the uncharged hydrogen atom, which cannot benefit from the Grotthuss effect, is nearly as high., The question is what mechanism can permit such a fast diffusion for this species?

In dilute gases, diffusion scales as the inverse square root of the mass, while in solids the mass effect depends on the diffusion mechanism and can either increase or decrease with particle mass. In liquids there are very few cases where a significant mass effect has been found, despite many attempts to find such a dependence; ${ }^{5}$ a convincing example is liquid ${ }^{6} \mathrm{Li} /{ }^{7} \mathrm{Li}$, where the lighter isotope diffuses faster by ca. $6 \% .{ }^{6}$

The vast majority of liquid diffusion data has been interpreted using the Stokes-Einstein equation for macroscopic spheres with a hydrodynamic radius $R_{0}$ in a liquid with viscosity $\eta$

$$
D=\frac{k T}{n \pi \eta R_{0}}
$$

where $k$ is the Boltzmann constant, $T$ is the absolute temperature, and $n$ takes values that vary from 6 in the "no- slip" limit, which applies to molecules which are large compared with solvent molecules, to 4 in the "slip" limit for solute and solvent molecules of comparable size. ${ }^{5}$ Note that eq 1 does not 4 contain the solute mass.

In this study we are interested in the diffusion of hydrogen atoms in water. On the basis of the work of Benderskii and Krivenko, ${ }^{4}$ who report a value of $D_{\mathrm{H}}=(7.7 \pm 1.0) \times 10^{-9} \mathrm{~m}^{2} \mathrm{~s}$ ${ }^{-1}$ at $298 \mathrm{~K}$, eq 1 yields a hydrodynamic radius $R_{0}=57 \mathrm{pm}$ (using $n=4$ ), which coincides with the atomic Bohr radius. Considering the soft radial decay of the 1 s wave function, such a small $R_{0}$ value cannot be regarded as physically meaningful and should instead be taken as an indication of the inapplicability of the Stokes-Einstein equation. Molecular dynamics simulations $^{7-12}$ and model fitting of EPR hyperfine coupling constants ${ }^{13}$ predict that $\mathrm{H}$ atoms in water reside in a cavity or "bubble", which has a radius of between 290 and 400 pm, ${ }^{12,13}$ much larger than the calculated $R_{0}$ value. If $\mathrm{H}$-atom diffusion occurred by a mechanism in which this bubble moves like a Stokes sphere, with corresponding hydrodynamic friction, 
one would predict diffusion coefficients of ca. $1 \times 10^{-9} \mathrm{~m}^{2} \mathrm{~s}^{-1}$. This is much smaller than experiment, indicating again, and not unexpectedly for such small particles, that the Stokes-Einstein relation breaks down.

So what is the diffusion mechanism for hydrogen atoms in water? A Car-Parrinello simulation by Kirchner et al. ${ }^{12}$ finds that $\mathrm{H}$-atom diffusion is driven by fluctuations of the hydrogenbonding network at the surface of the hydrophobic cavity, such that the light atom simply "follows" the diffusion of the cavity. The study also speculates that small hydrophobic species may simply populate the cavities that are naturally present in water, even in the absence of the solute. These vacant cavities are of very similar size and structure to the $\mathrm{H}$-atom hydrophobic solvation cavities, and they diffuse by a structural defect mechanism that is characterized by a fast exchange of water molecules at the cavity surface. ${ }^{14}$ After only 1.4 ps on average, all water molecules near the surface have exchanged, in contrast with small cations that travel for many picoseconds with their hydration shell attached. ${ }^{15}$ A diffusion constant of $D_{\mathrm{H}}=7.7 \times$ $10^{-9} \mathrm{~m}^{2} \mathrm{~s}^{-1}$ at $315 \mathrm{~K}$ was calculated for the hydrogen atom in BLYP water, in excellent agreement with the experimental result, and double the value that is obtained for the selfdiffusion of the water molecules. ${ }^{12}$

This was the starting point of the present investigation, which aims to test the model by comparing the rate constants of a diffusion-controlled process of the three hydrogen isotopes $\mathrm{D}, \mathrm{H}$, and $\mathrm{Mu}$ with masses of 2,1 , and $1 / 9 \mathrm{Da}$, respectively. $\mathrm{Mu}$ stands for muonium, a hydrogen-like one-electron atom with a positive muon as its nucleus. ${ }^{16}$ To within $0.5 \%$ it has the same Bohr radius and binding energy as the other isotopes so that its chemical properties are virtually the same, except when they depend on mass. We chose the diffusion-controlled "reaction" of these three species with $\mathrm{Ni}^{2+}$, which exists as a hexa-aquo complex in water: ${ }^{15}\left(\mathrm{Ni}^{2+}\right)_{\mathrm{aq}}=\mathrm{Ni}\left(\mathrm{H}_{2} \mathrm{O}\right)_{6}{ }^{2+} \cdot \mathrm{Ni}^{2+}$ does not react chemically with $\mathrm{H}$ atoms, as evidenced by the upper limit of $<3$ $\times 10^{5} \mathrm{M}^{-1} \mathrm{~s}^{-1}$ for a corresponding rate constant; ${ }^{17}$ however, the ion is paramagnetic with a spin of $S=1$, so that it undergoes spin exchange with hydrogen atoms, a process that is usually assumed to be nonactivated and diffusion-limited. ${ }^{18} \mathrm{We}$ note that Ghandi et al. made a similar choice to study a diffusion-limited process of muonium atoms in high-temperature pressurized water. ${ }^{19}$

Publication of the work in this manuscript was greatly delayed, and in the meantime Markland et al. have published a simulation study of the isotope effects on $\mathrm{H}, \mathrm{D}$, and $\mathrm{Mu}$ diffusion in water and ice using a classical TIP4P/2005 water model. ${ }^{10}$ Nuclear motion of the atomic hydrogen isotopes was treated quantum mechanically using ring polymer molecular dynamics (RPMD). By varying the mass of both the hydrogen atoms and the water molecules, these workers were able to analyze the diffusion in terms of both the cavity diffusion proposed by Kirchner et al., ${ }^{12}$ and an additional "hopping" mechanism previously proposed by Benderskii and coworkers, ${ }^{3,4}$ in which the light atom jumps between the adjacent natural cavities in the solution. A major quantum mechanical effect on the diffusion is the increasing de Broglie wavelength of the atom as the mass decreases. Markland et al. predict that the muonium atom will actually diffuse slower than the $\mathrm{H}$ or $\mathrm{D}$ atoms at room temperature because its de Broglie wavelength (3.0 ̊) is slightly larger than the natural water cavities. ${ }^{10}$ The present study can therefore be considered to be a test of these predictions.
In the following sections we describe the pulse radiolysis/ pulsed EPR experimental method and then describe the results using global data analysis, which improves upon previous methodology. The $\mathrm{Ni}^{2+}$ experiments were actually undertaken to improve on previous results with triplet $\mathrm{O}_{2}$, which had been published only as a conference proceedings ${ }^{20}$ (available online). The $\mathrm{Ni}^{2+}$ and $\mathrm{O}_{2}$ spin exchange rates, combined with reaction rates for the $\mathrm{H}$ atom self-recombination, ${ }^{21}$ are used in a discussion to deduce a self-consistent set of diffusion coefficients for the $\mathrm{H}, \mathrm{D}$, and $\mathrm{Mu}$ isotopes. The experimental results are tested with some additional RPMD simulations using a quantized/flexible water model.

\section{Experiments}

Experiments were carried out with dilute deoxygenated aqueous solutions in neat $\mathrm{H}_{2} \mathrm{O}$ or $90 \% \mathrm{D}_{2} \mathrm{O} / 10 \% \mathrm{H}_{2} \mathrm{O}$ solvent. Upon electron beam irradiation with $12-55 \mathrm{~ns}$ pulses of $3 \mathrm{MeV}$ electrons at $120 \mathrm{~Hz}$ from a van de Graaff accelerator, one generates $(\mathrm{e}-)_{\mathrm{aq}}, \mathrm{H}$ and/or $\mathrm{D}$ atoms, and $\mathrm{OH}$ radicals. ${ }^{22,23}$ The 1 solutions were adjusted to $\mathrm{pH} 2.0$ by the addition of perchloric acid to convert the dominant (e-) aq product to $\mathrm{H}$ and/or $\mathrm{D}$ atoms within ca. $10 \mathrm{~ns}$. The irradiation is performed in situ within the cavity of a pulsed X-band electron paramagnetic resonance (EPR) spectrometer. The $\mathrm{H}$ and $\mathrm{D}$ atoms are detected by measuring their free induction decay (FID) following a ca. $30 \mathrm{~ns} \pi / 2$ microwave pulse at the appropriate magnetic field settings. ${ }^{24}$ In the present experiments the $\mathrm{H}$ atom high field $\left(m_{\mathrm{I}}=-1 / 2\right)$ and $\mathrm{D}$-atom high field $\left(m_{\mathrm{I}}=-1\right)$ transitions were used. Examples of raw data can be seen in Figure 1 of the Results section and also in the previous publications. $^{20,25-28}$

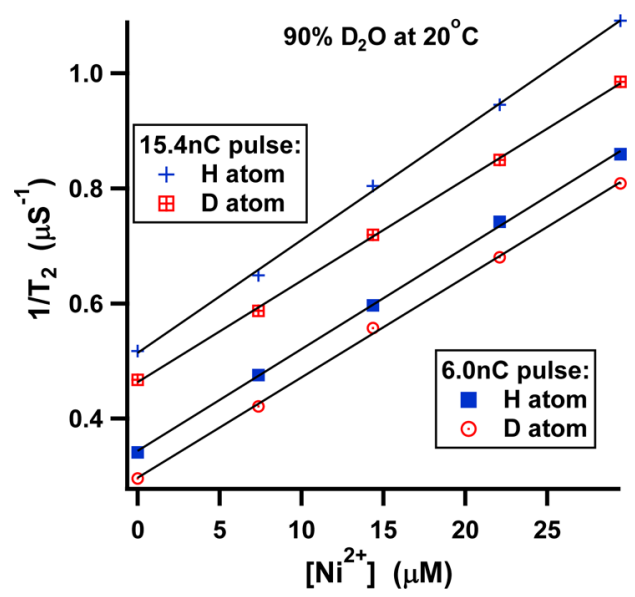

Figure 1. Effective single-exponential FID damping rate versus $\mathrm{Ni}^{2+}$ concentration in $90 \% \mathrm{D}_{2} \mathrm{O}$ at $20.1^{\circ} \mathrm{C}$.

The concentration dependence of the FID damping rate has been routinely measured for the determination of absolute chemical rate constants with an accuracy of a few percent. ${ }^{25-28}$ In general, a flow system recirculates several hundred milliliters of solution from a reservoir that is kept in a temperaturecontrolled bath. The concentration of "reactant" (in this case $\left(\mathrm{Ni}^{2+}\right)_{\mathrm{aq}}$, which does not react but causes spin relaxation) is changed by successive injections of additional reactant from a concentrated stock solution. The pseudo-first-order damping rate is plotted versus reactant concentration to extract the rate constant from the slope, as illustrated in Figure 1. In the several $\mathrm{kGy} /$ pulse radiation dose range typically used for good $\mathrm{S} / \mathrm{N}$ 
ratio, the slope depends slightly on the $\mathrm{H}$-atom concentration due to mutual spin exchange of the radicals, so a small correction is made for the second-order spin exchange by extrapolating to the "zero dose" limit. For the present experiment, the data analysis procedure was refined to further reduce potential systematic errors, as will be described in the Results section.

The present study follows a previous study ${ }^{20}$ of $\mathrm{H}$ and $\mathrm{D}$ atom spin exchange induced by collisions with triplet $\mathrm{O}_{2}$. In that study the radiation dose had to be carefully limited to avoid depleting the $\mathrm{O}_{2}$ scavenger concentration, and this limited the available $\mathrm{S} / \mathrm{N}$ ratio. The data were published as a conference proceedings (a complete description of the procedure is available online ${ }^{20}$ ) but never in the general literature, as we were not convinced of the $\mathrm{H} / \mathrm{D}$ isotope effect on the activation energy in $90 \% \mathrm{D}_{2} \mathrm{O}$.

The signal amplitudes of the EPR experiment are strongly affected by chemically induced dynamic electron polarization (CIDEP) from second-order recombination, ${ }^{29}$ both in radiation spurs $^{30,31}$ and in bulk. ${ }^{32,33}$ In previous work we found that very similar (absolute) signal amplitudes from $\mathrm{H}$ and $\mathrm{D}$ atoms are obtained in $90 \% \mathrm{D}_{2} \mathrm{O}$ thanks to the several relevant isotope effects. ${ }^{34}$ To study all three isotopes under identical solvent conditions the $\mathrm{Mu}$ experiments were conducted using muon irradiation of $\mathrm{Ni}^{2+}$ in $90 \% \mathrm{D}_{2} \mathrm{O}$ solvent as well. The experiment, which has been previously described, ${ }^{16}$ also measures the damping of a FID signal. It is therefore quite analogous to the EPR experiment, and the technique is called MuSR. The kinetics are ideally pseudo-first-order so that the experimental damping rate $\lambda_{\exp }$ of the FID is given by

$$
\lambda_{\exp }=\lambda_{0}+k_{\mathrm{ex}}\left[\mathrm{Ni}^{2+}\right]
$$

where $\lambda_{0}$ is a background relaxation and $k_{\mathrm{ex}}$ is the rate constant of electron spin exchange, which is obtained from the slope of a plot of $\lambda_{\exp }$ against concentration. In general, five concentrations of nickel perchlorate were used for each scavenging plot in this experiment. Sufficient counts were accumulated to obtain spin exchange rate constants of $\sim 5 \%$ precision or better. Because the electron spin exchange is relayed by the muon (nuclear) spin relaxation in low magnetic fields, the effective MuSR relaxation rate is actually a factor of 2 slower than the EPR relaxation, ${ }^{35}$ so that the observed MuSR values have to be doubled to obtain $k_{\mathrm{ex}}$.

\section{Results}

Figure 1 demonstrates a very typical pseudo-first-order "scavenging" plot for $\mathrm{H}$ and $\mathrm{D}$ atom dephasing by $\mathrm{Ni}^{2+}$ in $90 \% \mathrm{D}_{2} \mathrm{O}$ solution. The $30 \mathrm{~ns} \pi / 2$ microwave pulse is applied immediately following a 25 or 55 ns electron pulse, corresponding to kinetics delay $t=$ "zero". (The $t=$ zero delay is necessary in reaction rate measurements because the scavenger irreversibly removes the atomic hydrogen.) The free induction decays, damped cosines with period on the order of $100 \mathrm{~ns}$, are fit using single-exponential damping functions. The damping rate is plotted versus scavenger concentration. Figure 1 illustrates three qualities generally observed throughout the present experiment. The zero $\left[\mathrm{Ni}^{2+}\right]$ intercept of the plots depends on the radiation dose or initial radical concentration due to the second-order spin exchange with other $H, D$, and $\mathrm{OH}$ radicals. For given dose, the intercept for $\mathrm{H}$ atom is larger than that for $\mathrm{D}$ atom because the second-order spin-exchange rate for $\mathrm{H}$ is greater than that for $\mathrm{D}$. Finally, the slope of the lines for $\mathrm{H}$ atom depends slightly on the dose, but the $\mathrm{D}$-atom slopes are more nearly constant. This can also be ascribed to the greater second-order spin exchange of $\mathrm{H}$ relative to $\mathrm{D}$. The change in slope occurs because the total radical concentration is actually changing significantly on the time scale of the FID.

In the present experiment the $\mathrm{H}$ - and $\mathrm{D}$-atom concentrations do not actually change due to encounters with the "reactant" $\mathrm{Ni}^{2+}$. As a result, better $\mathrm{S} / \mathrm{N}$ ratio can be obtained for time delays $t>0$. Figure 2 illustrates FID shapes and amplitudes for

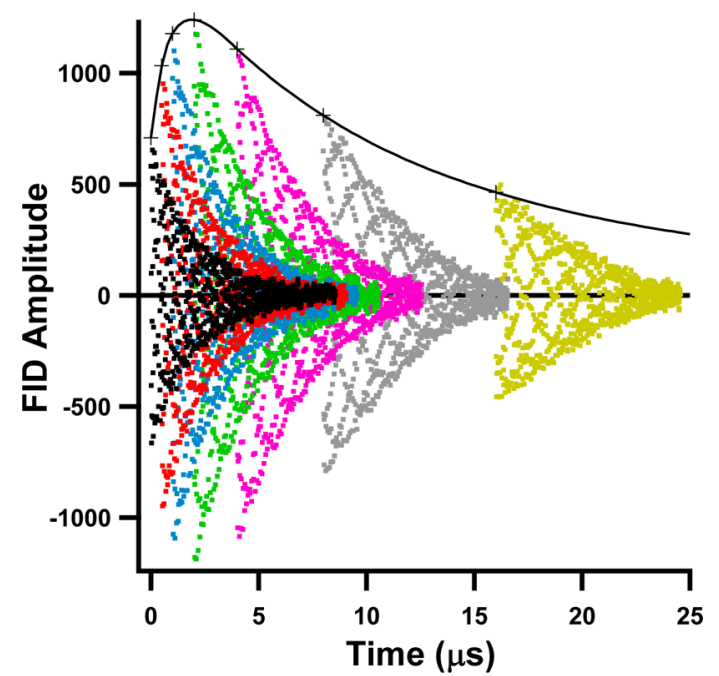

Figure 2. FIDs versus delay time after the electron pulse for the $D$ atom high field $\left(m_{\mathrm{I}}=-1\right)$ line in heavy water. The progression of the CIDEP versus kinetic delay is indicated by the line interpolating initial amplitudes of the FIDs.

the $\mathrm{D}$ atom high field $\left(m_{\mathrm{I}}=-1\right)$ line in heavy water with no $\mathrm{Ni}^{2+}$ present, as a function of the kinetics delay time $t$ between the electron pulse and the microwave $\pi / 2$ probe pulse. The amplitude of these FIDs corresponds to the progression of the CIDEP polarization due to the underlying second-order recombination chemistry. Initial magnetization is present thanks to recombination events in radiolysis spurs. ${ }^{31}$ The polarization increases due to fast second-order recombination in the first two microseconds. Subsequently, the second-order reactions slow down and with it the generation of CIDEP polarization. Relaxation of the CIDEP polarization by secondorder spin exchange is predominantly responsible for the smaller amplitudes at longer time because the first-order $T_{1}$ for $\mathrm{H}$ and $\mathrm{D}$ atoms is very long. ${ }^{36}$

In Figure 2 the shapes of the FIDs at delay times $t=0$ and 16 $\mu$ s are visibly different. In Figure 3 we superimpose normalized FIDs at $t=0$ and $16 \mu$ s for the $\mathrm{D}$ atom in $90 \% \mathrm{D}_{2} \mathrm{O}$. The damping envelope at $16 \mu \mathrm{s}$ has a visible "Gaussian" character, while at $t=0$ the envelope displays second-order character. The Gaussian character is to be expected in a nonhomogeneous magnetic field due to the distribution of Larmor frequencies across the sample. It can be shown that for spin exchange in a free radical sample decaying by second-order kinetics the transverse spin relaxation damping will be characterized by the functional form ${ }^{32}$

$$
S(t, \tau)=\left(\frac{T_{c}}{T_{c}+t+\tau}\right)^{Z} \exp \left(-\tau / T_{2}\right) \exp \left(-\tau^{2} / \delta^{2}\right)
$$




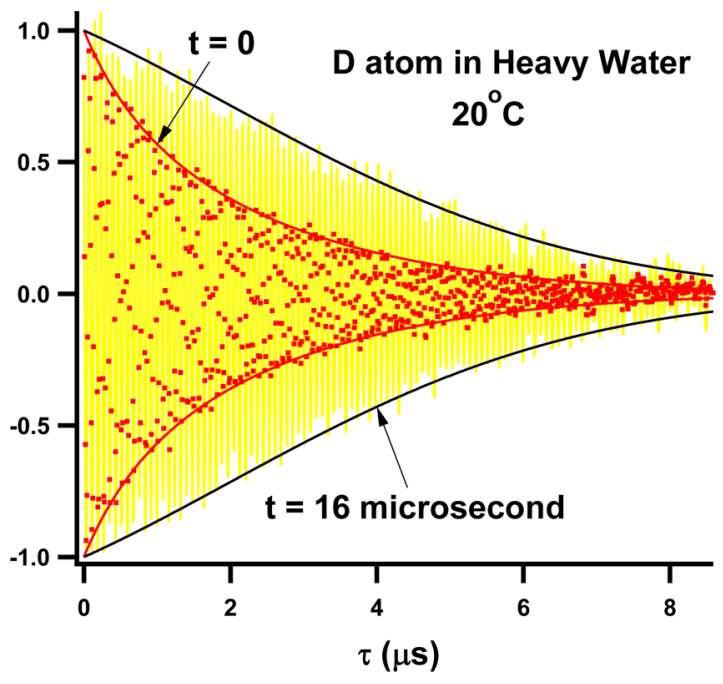

Figure 3. Normalized free induction decays of the $\mathrm{D}$ atom in $\mathrm{D}_{2} \mathrm{O}$, collected at delay times 0 and $16 \mu$ s following the electron radiolysis pulse. Lines indicate the fitted damping envelopes. For zero delay, the damping envelope is dominated by second-order spin exchange, while at $16 \mu \mathrm{s}$ the envelope is dominated by field inhomogeneity.

where $t$ is the kinetics delay as already defined, $\tau$ is the FID decay time measured from the $\pi / 2$ pulse, $T_{\mathrm{c}}$ is a chemical half life of the radical population measured from $t=0, T_{2}$ is the natural first-order decay time of transverse magnetization, and $\delta$ characterizes the field inhomogeneity. The exponent $Z=k_{\mathrm{ex}} /$ $k_{\mathrm{rxn}}$ is the ratio of second-order spin exchange and recombination rate constants. In general, $Z$ will be $>1.0$ because spin dephasing by Heisenberg exchange can occur at greater distances than those required to form a chemical bond. ${ }^{18,32}$

To thoroughly investigate the parameter space, we collected FIDs as in Figure 2 for both $\mathrm{H}$ atoms in light water and $\mathrm{H}$ and $\mathrm{D}$ atoms in $90 \% \mathrm{D}_{2} \mathrm{O}$ for two different radiation doses at 20,40, and $59{ }^{\circ} \mathrm{C}$. Each FID was prefit using an exponential damping function to precisely characterize its frequency, phase, and baseline offset. These parameters are virtually uncoupled from the shape of the damping envelope. For each temperature, the sets of 14 FIDs for different delay times and doses were then fit globally with eq 3 using the prefit information. The parameters $\mathrm{Z}$ and $T_{\mathrm{c}}$ are found to be very strongly covariant, so the constraint was imposed that the chemical half-life $T_{\mathrm{c}}$ is inversely proportional to the recorded charge/pulse of the electron beam (i.e., half life inversely proportional to initial radical concentration). The global fitting procedure was able to provide reasonably self-consistent parameters with completely random residuals.

The addition of $\mathrm{Ni}^{2+}$ to the aqueous samples has the intended effect of changing the first-order dephasing rate $1 / T_{2}$. For a series of temperatures between 10 and $60{ }^{\circ} \mathrm{C}$, free induction decays were recorded at delay time $t=0$ or $0.5 \mu \mathrm{s}$ using two radiation doses for both light water and $90 \% \mathrm{D}_{2} \mathrm{O}$. In addition to the water blank at $\mathrm{pH} 2, \mathrm{Ni}^{2+}$ was injected in four increments up to ca. $40 \mu \mathrm{M}$. The sets of $10 \mathrm{FIDs}$ (five concentrations, two doses) were fit globally using the same constraints as previously described, but now the effective firstorder dephasing rate is $k_{\text {ex }}\left[\mathrm{Ni}^{2+}\right]+1 / T_{2}$. The value of $\delta$ was fixed to an average value $\left(6.5 \times 10^{-6} \mathrm{~s}\right)$ found previously in the global fitting of neat heavy water. Values of $T_{2}$ were quite long, on the order of ten microseconds, and did not seem to follow a systematic trend with temperature. We suspect that these numbers are dominated by residual impurity and random noise. The chemical half-life $T_{c}$ has roughly the same Arrhenius behavior as the $\mathrm{Ni}^{2+}$ spin exchange, as expected for a diffusionlimited process, but with much greater scatter in the result. This no doubt comes from its covariance with the exponent $Z$, as noted above. The fitted values of $Z$ were in the range 1.1 to 1.3 .

The results are displayed in the form of Arrhenius plots of $k_{\text {ex }}$ in Figure 4, and Arrhenius parameters fitted to the raw data are

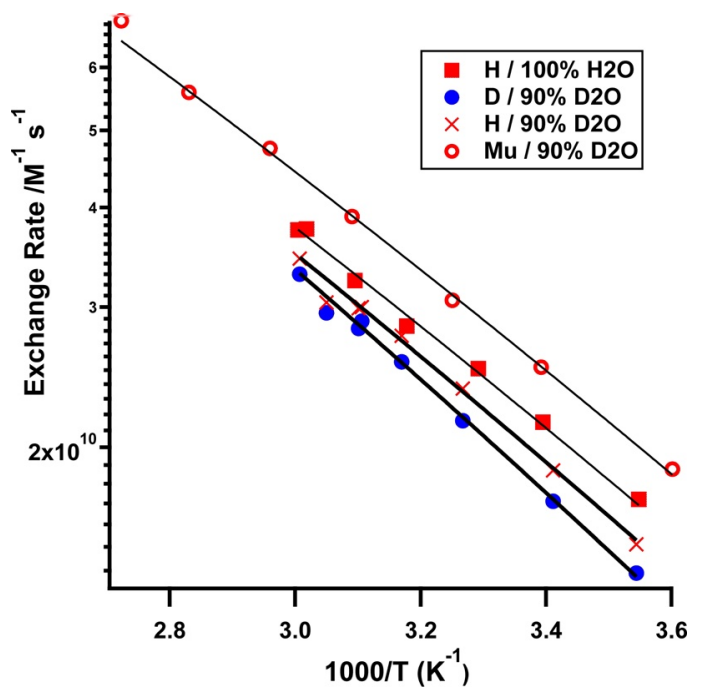

Figure 4. Spin-exchange rate constants for hydrogen isotopes $H, D$, and $\mathrm{Mu}$ with $\mathrm{Ni}^{2+}$ in light water and $90 \% \mathrm{D}_{2} \mathrm{O}$. Superimposed lines are from a model described in the text.

Table 1. Rate Constants and Arrhenius Parameters for Electron Spin Exchange of Hydrogen Isotopes with $\mathrm{Ni}^{2+}$

$\begin{array}{lccc}\text { reaction } & \begin{array}{c}k_{\mathrm{ex}}^{298 \mathrm{~K}} \\ \left(10^{10} \mathrm{M}^{-1} \mathrm{~s}^{-1}\right)\end{array} & \begin{array}{c}\log _{1^{0}} \\ \left(A / \mathrm{M}^{-1} \mathrm{~s}^{-1}\right)\end{array} & \begin{array}{c}E_{\mathrm{a}} \\ (\mathrm{kJ} \mathrm{mol}\end{array} \\ \mathrm{Mu}+\mathrm{Ni}^{2+} / 90 \% \mathrm{D}_{2} \mathrm{O} & 2.69 & 12.53(3) & 12.0(2) \\ \mathrm{H}+\mathrm{Ni}^{2+} / 90 \% \mathrm{D}_{2} \mathrm{O} & 2.04 & 12.44(7) & 12.2(4) \\ \mathrm{D}+\mathrm{Ni}^{2+} / 90 \% \mathrm{D}_{2} \mathrm{O} & 1.90 & 12.49(8) & 12.6(5) \\ \mathrm{H}+\mathrm{Ni}^{2+} / 100 \% \mathrm{H}_{2} \mathrm{O} & 2.21 & 12.43(5) & 11.9(3)\end{array}$

listed in Table 1. All four spin-exchange processes show good linearity in the Arrhenius plot. (However, the fit lines superimposed on the plot come from a model described later.) It is obvious from the plot that there is a mass effect of the atomic isotope, with $\mathrm{Mu}$ atoms undergoing $38 \%$ faster and $\mathrm{D}$ atoms $8 \%$ slower spin exchange than $\mathrm{H}$ at $298 \mathrm{~K}$ in the common $90 \% \mathrm{D}_{2} \mathrm{O}$ solvent. (These ratios are slightly temperature-dependent. The rate constant ratios supersede those of a previous determination based on spin exchange of $\mathrm{Mu}$ with $\mathrm{O}_{2}$, which had to be separated from chemical reaction and therefore was less accurate. ${ }^{37}$ ) A second isotope effect is present in comparison of the $\mathrm{H}$ atom diffusion in $90 \% \mathrm{D}_{2} \mathrm{O}$ to its diffusion in $100 \% \mathrm{H}_{2} \mathrm{O}$. This is not unexpected given the ca. $20 \%$ difference in viscosity of light and heavy water ${ }^{38}$ and ultimately represents a quantum effect of the water librations. ${ }^{39}$

\section{Discussion}

A. Activation Energy of H-Atom Diffusion. How can one measure the diffusion coefficient of a short-lived transient 
such as the hydrogen atom? The obvious way is to measure a diffusion-limited process involving the transient. In the present study and the previous $\mathrm{O}_{2}$ work, ${ }^{20}$ we measure diffusion-limited spin exchange because it is also possible to directly measure the isotope effect of the atoms independent of the solvent. According to the Smoluchowski equation for a diffusionlimited process involving encounters between spheres $\mathrm{A}$ and $\mathrm{B}^{40}$

$$
k_{\text {diff }}=4000 \pi N_{\mathrm{A}} \sigma R_{\mathrm{eff}}\left(D_{\mathrm{A}}+D_{\mathrm{B}}\right)
$$

$k_{\text {diff }}$ (in units of $\mathrm{M}^{-1} \mathrm{~s}^{-1}$ where $N_{\mathrm{A}}$ is the Avogadro constant) is proportional to the sum of the diffusion coefficients, $D_{\mathrm{A}, \mathrm{B}}$, of the two encounter partners. (It is important to note that if $\mathrm{A}$ and $\mathrm{B}$ are identical particles $k_{\text {diff }}$ needs to be multiplied by $1 / 2$ to avoid double-counting encounters.) To extract the desired information on the hydrogen diffusion coefficient, we need to know about the diffusion of the exchange partner, establish the spin statistical factor $\sigma$, and define the reaction distance, $R_{\text {eff }}$. In the most basic Smoluchowski model for diffusion-limited encounters, $R_{\text {eff }}$ is simply the contact distance, equal to the sum of radii of two diffusing spheres. Spin-exchange dephasing requires that the strength (expressed as angular frequency) of Heisenberg exchange $J$ integrated over the duration of the encounter $\tau$ should be greater than unity, $J^{*} \tau>1 .^{18}$ While this spin-dephasing process is usually diffusion limited, there is a complication in defining the effective reaction distance. It is well-established that spin dephasing can occur via "weak" encounters in which the respective particles do not suffer a "hard" collision. Assuming that the Heisenberg spin exchange falls off exponentially with distance from the "hard sphere encounter distance", an effective reaction radius for the strong exchange limit has been derived by Molin et al. ${ }^{18}$ based on a diffusive first passage approximation

$$
R_{\mathrm{ex}}=r_{\mathrm{ab}}+r_{\mathrm{o}} \ln \left(J_{\mathrm{o}} r_{\mathrm{o}}^{2} / D\right)
$$

Here $D$ is the relative diffusion $\left(D_{\mathrm{a}}+D_{\mathrm{b}}\right), J_{\mathrm{o}}$ is exchange frequency at the encounter distance $r_{\mathrm{ab}}$, and $r_{\mathrm{o}}$ characterizes the falloff of the exchange: $J(r)=J_{\mathrm{o}} \exp \left(-\left(r-r_{\mathrm{ab}}\right) / r_{\mathrm{o}}\right)$. The implication of this formula is that $R_{\mathrm{ex}}$ will increase when diffusion is slow, and so it must increase at lower temperature. It means that the observed activation energy of spin exchange, as found in Table 1, represents a lower limit for the actual diffusional activation energy.

A more straightforward experiment for isotopically pure solvent is simply to measure the diffusion-limited second-order recombination of $\mathrm{H}$ atoms. This has been done many years ago by Sehested and Christensen in a transient absorption measurement using the absorbance attributed to the $\mathrm{H}$ atom in the deep UV below $250 \mathrm{~nm} .^{21}$ (It is universally agreed that this absorbance is due to photoinduced charge transfer from water solvent into the hydrogen atom 1 s orbital.) The secondorder kinetics information was reported in the form of $2 k_{\mathrm{HH}}$ / $\varepsilon L$, where $\varepsilon L$ is the product of extinction coefficient and optical path length. Sehested and Christensen recommend the temperature-independent value $\varepsilon=950 \mathrm{M}^{-1} \mathrm{~cm}^{-1}$ at $200 \mathrm{~nm}$ to extract the rate constants but suggest that this number may increase on the order of $10-15 \%$ by $200{ }^{\circ} \mathrm{C}$. The average Arrhenius activation energy for this "raw" reaction rate data is $14.7 \mathrm{~kJ} / \mathrm{mol}$, which according to the authors should represent a lower limit. ${ }^{21}$ In Figure 5 we plot the $2 k_{\mathrm{HH}}$ rate constant data collected by Sehested and Christensen ${ }^{21}$ up to $250{ }^{\circ} \mathrm{C}$, along with the light water $\mathrm{O}_{2}$ data collected in our previous work ${ }^{20}$

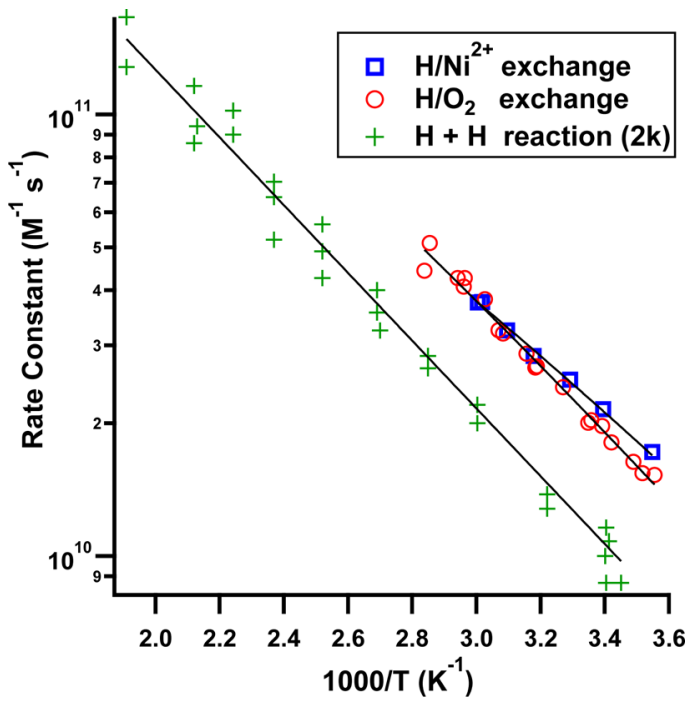

Figure 5. Rate constants for self-recombination $\left(2 k_{\mathrm{HH}}\right)$ of $\mathrm{H}$ atoms, spin exchange of $\mathrm{H}$ with $\mathrm{Ni}^{2+}$, and spin exchange/reaction of $\mathrm{H}$ with $\mathrm{O}_{2}$ in light water.

and the $\mathrm{Ni}^{2+}$ data collected in the present report. It can be seen immediately that the $\mathrm{O}_{2}$ spin exchange has nearly the same activation energy as the $\mathrm{H}$ recombination reaction, while the $\mathrm{Ni}^{2+}$ exchange has slightly lower activation energy. Elliot and Bartels reviewed the $\mathrm{H}$ recombination data along with all of the others involved in water radiolysis in $2008 .^{23}$ These reviewers 3 accepted that the $\mathrm{H}$-atom extinction coefficient might increase slightly, and the best estimate of activation energy is $15.5 \mathrm{~kJ} /$ mol, with room temperature value $2 k_{\mathrm{HH}}=1.2 \times 10^{10} \mathrm{M}^{-1} \mathrm{~s}^{-1}$. In the present work, we accept this number for room temperature but retain the $14.7 \mathrm{~kJ} / \mathrm{mol}$ activation energy because it fits slightly better to the muonium data in the treatment below.

The recent $\mathrm{MD}$ modeling studies of Pomogaeva and Chipman, ${ }^{9}$ based on high-level ab initio $H$ versus water potentials and the high-quality AMOEBA polarizable water model, ${ }^{41-43}$ establish that the average "cage" of water molecules around the $\mathrm{H}$ atom will be at a (oxygen) distance $g_{\mathrm{HO}}(r)=3.0 \AA$ independent of temperature in the liquid density regime. For two $\mathrm{H}$ atoms to react, they need to diffuse together into the same solvent cage. We can confidently choose the reaction distance $R_{\text {eff }}=3.0 \AA$ as the barrier that must be passed for this reaction. (The $\mathrm{H}$-atom van der Waals radius of $\sim 1 \AA$ is not limiting. Recall according to the Smoluchowski boundary condition, $R_{\text {eff, }}$ is the distance at which reaction is certain to occur. Once a pair of $\mathrm{H}$ atoms occupy the same cavity, reaction must be certain.) As we have already noted, the natural spin relaxation of $\mathrm{H}$ atom is very long, so only $1 / 4$ of the encounters (singlet) will result in reaction. Taking the room-temperature value of $2 k_{\mathrm{HH}}=1.2 \times 10^{10} \mathrm{M}^{-1} \mathrm{~s}^{-1}$, we obtain diffusion coefficient of the $\mathrm{H}$ atom as $1.0 \times 10^{-8} \mathrm{~m}^{2} \mathrm{~s}^{-1}$. We estimate no more than $20 \%$ error in this number given the scatter in the transient absorption experiment. ${ }^{21}$

Using this calibration of the diffusion coefficient from the reaction rate data, we can apply the first passage formula to fit the more precise spin exchange data. The $\mathrm{O}_{2}$ diffusion coefficient versus temperature is known from direct measurements made using the Taylor dispersion (HPLC linebroadening) technique. ${ }^{44}$ Diffusion of $\mathrm{Ni}^{2+}$ is measured at 4 room temperature by its specific conductivity and the Nernst- 
Einstein equation: $D_{\mathrm{Ni}}=1.41 \times 10^{-9} \mathrm{~m}^{2} \mathrm{~s}^{-1}$. The hydrated ion moves along with its hydration layer of six water molecules, ${ }^{15}$ so we can assume the validity of the Stokes-Einstein formula and scale the diffusion by $T /$ viscosity. Using these diffusion coefficients, we can now evaluate the effective distance for spin exchange. Assuming the statistical factor $\sigma=16 / 27$ for triplet dephasing of a doublet, ${ }^{18,35} R_{\mathrm{ex}}=3.50 \AA$ for $\mathrm{O}_{2}$ and $R_{\mathrm{ex}}=3.99$ $\AA$ for $\mathrm{Ni}^{2+}$ at room temperature.

Fits of the spin-exchange rate using eq 5 are superimposed on the light water data in Figure 5. For $\mathrm{O}_{2}$, the activation energy, similar to that of the recombination reaction, implies that the spin exchange is very short-ranged. The best fit gives $r_{\mathrm{o}}$ $=0.20 \AA$ and $J_{\mathrm{o}}=2.6 \times 10^{17} \mathrm{~s}^{-1}$ for $r_{\mathrm{ab}}=2 \AA$. No good fit can be obtained for $r_{\mathrm{o}}>0.4 \AA$. Choice of the hard sphere encounter distance between 2.0 and $3.0 \AA$ makes no difference to the fit because the parameters are covariant with the spin-exchange $J_{\mathrm{o}}$. $\mathrm{O}_{2}$, like the $\mathrm{H}$ atom, is a small hydrophobe whose diffusion does not obey the Stokes-Einstein relation. ${ }^{20}$ The spinexchange encounters may be strongly modulated by the water caging effect, such that exchange is either "on" or "off". This might produce the short effective exchange distance from the fit. This same caging phenomenon might explain the relatively small value of $Z=k_{\mathrm{ex}} / 2 k_{\mathrm{HH}}$ found in analyzing the $\mathrm{H}$ and $\mathrm{D}$ atom FID data.

The activation energy for $\mathrm{Ni}^{2+}$ spin exchange is slightly smaller than for $\mathrm{O}_{2}$, and the exchange is apparently longerranged. The second hydration shell peak of Ni versus $\mathrm{O} g(r)$ in $\mathrm{X}$-ray scattering measurements is found at ca. $4.0 \AA .^{15}$ Assuming that the water oxygen van der Waals radius is $1.6 \AA$ while the $\mathrm{H}$-atom radius is $1.0 \AA$, we choose $r_{\mathrm{ab}}=3.4 \AA$ as the hardsphere contact radius between $\mathrm{Ni}^{2+}$ and the $\mathrm{H}$ atom. The fit of the light water data to eq 5 shown in Figure 5 gives $J_{\mathrm{o}}=5.30 \times$ $10^{12} \mathrm{~s}^{-1}$ and $r_{\mathrm{o}}=0.847 \AA$. This falloff parameter is quite typical for spin exchange between atomic species. ${ }^{18}$

It is worth remarking that our use of eq 5 assumes a "strong exchange" limit for $\mathrm{H}$ versus $\mathrm{Ni}^{2+}$ encounters to explain the difference in activation energies of the $2 k_{\mathrm{HH}}$, oxygen exchange, and $\mathrm{Ni}^{2+}$ exchange data. This also requires the use of $16 / 27$ statistical factor, which may be questionable in the presence of fast spin relaxation of the metal complex. ${ }^{45}$ If we assume fast spin relaxation and use $\sigma=1.0$ for the spin statistical factor, the effective exchange distance becomes $R_{\text {eff }}=2.36 \AA$. If we then assume the probability for exchange is only $70 \%$ per encounter, we could explain the room temperature number in terms of weak exchange. ${ }^{18}$ Ghandi et al. ${ }^{19}$ have reported a maximum in spin exchange between $\mathrm{Mu}$ and $\mathrm{Ni}^{2+}$ near $130{ }^{\circ} \mathrm{C}$, which they interpret in terms of weak exchange, as the duration of encounters decreases at high temperature; however, our attempts to use equations appropriate to the weak exchange situation near room temperature fail to quantitatively explain the $\mathrm{Ni}^{2+}$ activation energies. We conclude that spin relaxation of $\mathrm{Ni}^{2+}$ is not actually so fast that it dominates the dephasing.

With the spin-exchange parameters for $\mathrm{Ni}^{2+}$ determined in light water, we are in a position to evaluate the actual $\mathrm{H}, \mathrm{D}$, and $\mathrm{Mu}$ diffusion coefficients in the $90 \% \mathrm{D}_{2} \mathrm{O}$ experiments. We make the very minor correction that diffusion of $\mathrm{Ni}^{2+}$ is slightly slower by the ratio of $T /$ viscosity in $90 \% \mathrm{D}_{2} \mathrm{O}$ versus $\mathrm{H}_{2} \mathrm{O}$. The data for $\mathrm{H}, \mathrm{D}$, and $\mathrm{Mu}$ were then fit very well, as illustrated by the lines in Figure 4. Fitted Arrhenius parameters for the diffusion of $\mathrm{H}, \mathrm{D}$, and $\mathrm{Mu}$ in $90 \% \mathrm{D}_{2} \mathrm{O}$ are collected in Table 2. The fitted activation energies for diffusion are several kilojoules per mole larger than the numbers deduced directly from the spin-exchange data and are also higher than the $14.7 \mathrm{~kJ} / \mathrm{mol}$
Table 2. Arrhenius Parameters for Hydrogen Isotope Diffusion Deduced in This Work

$\begin{array}{cccc}\text { isotope/solvent } & D^{298 \mathrm{~K}}\left(10^{-8} \mathrm{~m}^{2} \mathrm{~s}^{-1}\right) & A / 10^{-6} \mathrm{~m}^{2} \mathrm{~s}^{-1} & E_{\mathrm{a}}\left(\mathrm{kJ} \mathrm{mol}{ }^{-1}\right) \\ \mathrm{Mu} / 90 \% \mathrm{D}_{2} \mathrm{O} & 1.29 & 5.26 \pm 0.46 & 14.9 \pm 0.2 \\ \mathrm{H} / 90 \% \mathrm{D}_{2} \mathrm{O} & 0.901 & 4.36 \pm 0.50 & 15.3 \pm 0.3 \\ \mathrm{D} / 90 \% \mathrm{D}_{2} \mathrm{O} & 0.802 & 6.23 \pm 0.90 & 16.5 \pm 0.4 \\ \mathrm{H} / 100 \% \mathrm{H}_{2} \mathrm{O} & 1.06 & 3.75 \pm 0.95 & 14.7 \pm 1.0\end{array}$

activation energy in light water. They remain lower than the Stokes-Einstein activation energy of $T /$ viscosity.

The foregoing analysis begins with the $2 k_{\mathrm{HH}}$ reaction rate constants, which unfortunately have the largest experimental uncertainty. The scatter of the numbers at a given temperature is on the order of $25 \%$, and some systematic error may be present due to error in the extinction coefficients $\varepsilon$ used to convert the observable $2 k_{\mathrm{HH}} / \varepsilon L$ into the rate constant. The data have been reviewed, ${ }^{23}$ and it seems unlikely that the systematic error could be more than several percent at room temperature. At very high temperature, it is suspected that the extinction coefficient of the optical transition increases, which means the activation energy of the reaction would be larger. ${ }^{23}$ Recall that the more precise spin exchange data will give a lower limit of the activation energy. We tend to believe the activation energy of the reaction rate data because the $\mathrm{O}_{2}$ spin-exchange data cannot be explained otherwise. The uncertainty in absolute light water diffusion coefficient cannot possibly be more than $\sim 20 \%$.

Measurement of $\mathrm{H}$ and $\mathrm{D}$ atom diffusion coefficient was reported previously by Benderskii and Krivenko, ${ }^{4}$ who photoejected electrons from a mercury drop electrode into acidic electrolyte using a nanosecond UV laser pulse. The solvated electrons will return to the metal surface but may first react with acid protons to give $\mathrm{H}$ atoms. By time-resolving the current from this photoejection transient, they were able to extract the relative diffusion rates of $\mathrm{H}$ atom and $(\mathrm{e}-)_{\mathrm{aq}}$ in strong $(0.1$ to $2.0 \mathrm{M})$ electrolyte solution. The $(\mathrm{e}-)_{\text {aq }}$ diffusion back to the surface could be measured directly in the absence of acid. An extrapolation procedure was then required to estimate the diffusion coefficients in dilute solution. While this experiment certainly gives the correct order-of-magnitude $\left(D_{\mathrm{H}}=7.7 \times 10^{-9} \mathrm{~m}^{2} \mathrm{~s}^{-1}\right.$ at room temperature $\left.{ }^{4}\right)$, we believe the overall procedure is subject to a number of systematic errors. The activation energies for both $\mathrm{H}$ in light water and $\mathrm{D}$ in heavy water were reported as $12 \pm 1.5 \mathrm{~kJ} / \mathrm{mol}$, which is incompatible with the present spin-exchange data and the reaction rate data.

The RPMD study of Markland et al. ${ }^{10}$ using TIP4P3/2005 water found a room-temperature diffusion coefficient of $9.9 \times$ $10^{-9} \mathrm{~m}^{2} \mathrm{~s}^{-1}$ for the $\mathrm{H}$ atom in perfect agreement with our estimate but with an activation energy of only $10 \mathrm{~kJ} / \mathrm{mol}$, which is certainly too low. The $\mathrm{H}$-atom study of Pomogaeva and Chipman' using the AMOEBA water model found a room temperature value of $8.8 \times 10^{-9} \mathrm{~m}^{2} \mathrm{~s}^{-1}$, probably within error of our higher result based on $2 k_{\mathrm{HH}}$. Their simulations found activation energy of $12.6 \mathrm{~kJ} / \mathrm{mol}$ up to $200{ }^{\circ} \mathrm{C}$, but the activation energy increased at higher temperature. The latter feature may agree with the reaction rate experiment of Sehested and Christensen if $\mathrm{H}$-atom extinction coefficient increases substantially at high temperature; ${ }^{23}$ however, activation energy $s$ in the lower temperature region is still slightly low. All of the recent $\mathrm{MD}$ studies agree that the first peak in the $\mathrm{H}$ versus water oxygen radial distribution function $g_{\mathrm{HO}}(r)$ should be 
found at $3.0 \AA$, which is the number needed to deduce the diffusion coefficient from the measured reaction rate.

B. Isotope Effects on the Diffusion. Markland et al. ${ }^{10}$ were able to decompose the (classical) isotope effects on $H, D$, and $\mathrm{Mu}$ atom diffusion into a linear combination of "cavity diffusion" $D_{\text {cav }}$ and "hopping" $D_{\text {hop }}$ between adjacent solvent cavities. The former mechanism is the same one proposed by Kirchner et al., ${ }^{12}$ while the latter mechanism was proposed by Benderskii and coworkers. ${ }^{3,4}$ Cavity diffusion should depend only on the water molecule mass, while the hopping frequency is proportional to the inverse square root of the isotopic hydrogen mass. Markland et al. propose the net diffusion coefficient can be written

$$
{ }_{8} \quad D(m, M)=\left(m_{\mathrm{H}} / m\right)^{1 / 2} D_{\text {hop }}+\left(M_{\mathrm{H}_{2} \mathrm{O}} / M\right)^{1 / 2} D_{\text {cav }}
$$

where $M$ is the mass of solvent and $m$ is the mass of the solute. On the basis of the RPMD simulations of Markland et al., the $\mathrm{H}$ and D diffusion should be essentially classical. Using our roomtemperature diffusion coefficients for $\mathrm{H}$ and for $\mathrm{D}$ in $90 \% \mathrm{D}_{2} \mathrm{O}$, we are able to deduce that $D_{\text {hop }}=3.38 \times 10^{-9} \mathrm{~m}^{2} \mathrm{~s}^{-1}$ and $D_{\text {cav }}=$ $5.63 \times 10^{-9} \mathrm{~m}^{2} \mathrm{~s}^{-1}$ for a ratio $D_{\text {hop }} / D_{\text {cav }}=0.60$. Using these values, we can now predict that the classical muonium diffusion coefficient in $90 \% \mathrm{D}_{2} \mathrm{O}$ should be $D_{\mathrm{Mu}}=(5.63+3 \times 3.38) \times$ $10^{-9} \mathrm{~m}^{2} \mathrm{~s}^{-1}$ or $D_{\mathrm{Mu}}=1.58 \times 10^{-8} \mathrm{~m}^{2} \mathrm{~s}^{-1}$. We find the experimental muonium diffusion is nearly as fast (80\%) as this classical prediction, at $1.29 \times 10^{-8} \mathrm{~m}^{2} \mathrm{~s}^{-1}$. There seems to be relatively little evidence of muonium slowing down dramatically due to the "quantum swelling" phenomenon predicted by Markland et al. ${ }^{10}$ Moreover, the diffusional activation energies of the muonium and hydrogen isotopes differ by only $0.4 \mathrm{~kJ} /$ mol as opposed to $2.2 \mathrm{~kJ} / \mathrm{mol}$ in the classical simulation. ${ }^{10}$ Within error, muonium appears to behave almost classically in the $90 \% \mathrm{D}_{2} \mathrm{O}$ solvent.

If we now consider the classical solvent isotope effect of eq 6, we can predict that in light water the cavity diffusion will be enhanced by a factor $\sqrt{ }(20 / 18)=1.05$, so the $\mathrm{H}$ atom diffusion ought to be $D_{\mathrm{H}}=(3.38+1.05 \times 5.63) \times 10^{-9} \mathrm{~m}^{2} \mathrm{~s}^{-1}$ or $D_{\mathrm{H}}=$ $9.29 \times 10^{-9} \mathrm{~m}^{2} \mathrm{~s}^{-1}$. This is only about a third of the solvent isotope effect found in the experiment. It is no great surprise that additional solvent isotope effect should be required. The difference in properties of light and heavy water are well known to be due to zero-point energy differences of the $\mathrm{H}_{2} \mathrm{O}$ versus $\mathrm{D}_{2} \mathrm{O}$ vibrations and librations, ${ }^{39}$ which is ultimately a quantum effect completely omitted from eq 6 and from the TIP4P/2005 simulations of Markland et al. ${ }^{10}$

Where is the dramatic quantum swelling effect on muonium diffusion predicted by the RPMD simulation ${ }^{10}$ and by the simple consideration of the de Broglie wavelength? The missing quantum isotope effects of the solvent prompted us to try an RPMD simulation of our own, using a quantized version of the TIP4P water model. Habershon et al. have demonstrated a qTIP4p/f quantum, flexible anharmonic water model, which does a very fine job of capturing the isotope effects on water density, diffusion coefficients, and infrared absorption frequencies when used with the RPMD method to simulate the nuclear motions. ${ }^{39}$ In addition to this change in the water potential, we wished to adopt the AMOEBA-style $\mathrm{H}$ atom versus water potential of Pomogaeva and Chipman ${ }^{9}$ because it was constructed from an in-depth ab initio study of the interaction as a function of geometry. Unfortunately the qTIP4p/f model is not 3-body polarizable, so we only keep the dominant spherically symmetric Lennard-Jones 14-7 term of ref 9. This correctly captures the average interaction and shallow attractive 606 well but not the $( \pm 0.2 \AA)$ asymmetry of approach to the "hard 607 wall”. The LJ 14-7 potential is compared in Figure 6 with the $608 \mathrm{fo}$

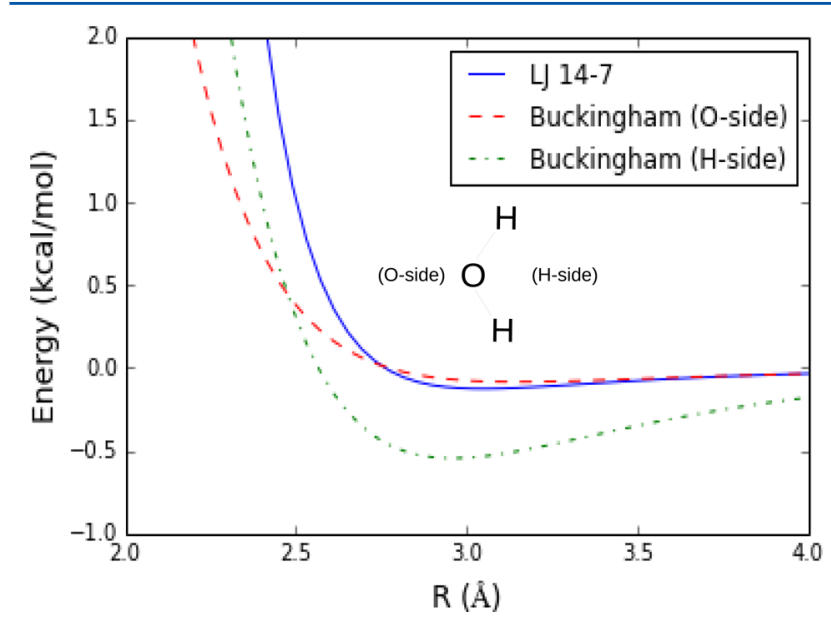

Figure 6. Comparison of $\mathrm{H}$ atom versus water (Buckingham) potential of Markland et al. ${ }^{10}$ with the LJ 14-7 potential used in this work.

empirical Buckingham potential used by Markland et al. for two directions of approach. The Buckingham potential is much more attractive at the hydrogen sites than is consistent with the ab initio calculations. ${ }^{\text {? }}$

Using the OpenMM software package, an RPMD simulation of hydrogen and muonium particles in a $36 \AA$ cubic box containing $1500 \mathrm{qTIP} 4 \mathrm{p} / \mathrm{f}$ water molecules at $300 \mathrm{~K}$ with 32 beads representing each hydrogen nucleus (water and solute) was stepped for $10 \mathrm{ps}$, taking 0.5 fs steps. Apart from using a larger box with more water molecules, the procedure used was identical to that described by Markland et al. ${ }^{10}$ The diffusion 6 coefficient was calculated using the Einstein relation: $6 t D=<1$ $r_{\mathrm{i}}(t)-\left.r_{\mathrm{i}}(0)\right|^{2}>$. The $g_{\mathrm{XO}}(r)$ and $g_{\mathrm{XH}}(r)$ functions for $\mathrm{X}=\mathrm{H}$ atom or muonium are shown in Figure 7.

Despite the steeper wall and spherical symmetry of the LJ 147 potential, the radial distributions in Figure 7 are virtually identical to those of Markland et al. and Pomogaeva and Chipman, ${ }^{9,12}$ with a maximum in $g_{\mathrm{HO}}(r)$ at $3.0 \AA$. Some effect of "quantum swelling" is obvious from the slight shift of the muonium $g_{\mathrm{MuO}}(r)$ to larger distance, again in good agreement with the classical TIP4P water simulation of Markland et al. The $\mathrm{H}$-atom diffusion coefficient we obtain, $D_{\mathrm{H}}=8.7 \times 10^{-9}$ $\mathrm{m}^{2} \mathrm{~s}^{-1}$, is in excellent agreement with both previous studies; ${ }^{9,10}$ however, calculation of the muonium diffusion rate produces a different result (see Table 3 ). The muonium diffuses faster with coefficient $D_{\mathrm{Mu}}=16.2 \times 10^{-9} \mathrm{~m}^{2} \mathrm{~s}^{-1}$ to give 1.8 for the ratio $D_{\mathrm{Mu}} / D_{\mathrm{H}}$, as opposed to 0.92 in classical TIP4P water. $^{10}$

The behavior of muonium diffusion in our simulation appears to be essentially the fully "classical" behavior found with the Buckingham potential by Markland et al. ${ }^{10}$ As a crosscheck we also ran some trajectories in the heavy water version of $\mathrm{qTIP} 4 \mathrm{p} / \mathrm{f}$ and obtained the same ratio for $D_{\mathrm{Mu}} / D_{\mathrm{H}}$ within the statistical error. What has happened to the inverse isotope effect on $\mathrm{Mu}$ diffusion, ascribed to inhibition of the "hopping" mechanism? Manolopoulos and coworkers reported to us ${ }^{46}$ that when they ran the Buckingham potential simulation in the qTIP4p/f water model they obtained nearly the same result as in classical TIP4p. The difference in our result is apparently due to the spherically symmetric and less-attractive LJ-14-7 

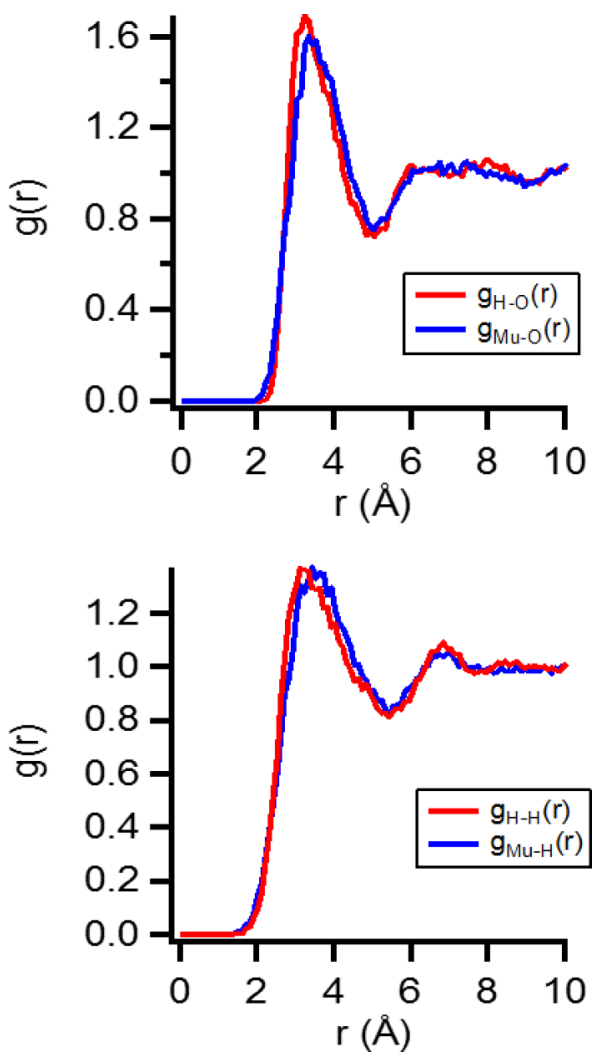

Figure 7. Radial distribution functions for $\mathrm{Mu}$ and $\mathrm{H}$ in liquid water at $300 \mathrm{~K}$. Top: $g_{\mathrm{XO}}(r)$. Bottom: $g_{\mathrm{XH}}(r)$.

Table 3. Diffusion Coefficients Determined from RPMD Simulations with qTIP4p/f Water Model and LJ 14-7 H Atom versus Water Potential

$\begin{array}{cccc}\text { particle } & \text { solvent } & D\left(10^{-9} \mathrm{~m}^{2} / \mathrm{s} \pm 95 \% \text { confidence }\right) & \text { no. } 10 \text { ps trials } \\ \mathrm{H} & \mathrm{H}_{2} \mathrm{O} & 8.7 \pm 0.7 & 100 \\ \mathrm{Mu} & \mathrm{H}_{2} \mathrm{O} & 16.2 \pm 1.9 & 100 \\ \mathrm{H} & \mathrm{D}_{2} \mathrm{O} & 8.4 \pm 1.6 & 20 \\ \mathrm{Mu} & \mathrm{D}_{2} \mathrm{O} & 17.0 \pm 4.5 & 20\end{array}$

potential (see Figure 6). One might expect the $g(r)$ distribution functions should reflect these differences in potential, but the $g(r)$ differences are minor. The muonium atom definitely is "larger" than the hydrogen atom by about the same amount in both simulations. It may be that the quantum swelling effect does in fact attenuate an even faster classical muonium hopping rate with the LJ-14-7 potential. We can only conclude that the muonium diffusion is much more sensitive to the details of the interaction potential than is the $\mathrm{H}$-atom diffusion. A better potential with intermediate steepness might reproduce the room-temperature experimental $D_{\mathrm{Mu}} / D_{\mathrm{H}}$ ratio of 1.4 and a correct activation energy. We leave it to others to investigate this effect with further simulations.

\section{Summary}

Diffusion of atomic hydrogen isotopes $\mathrm{D}, \mathrm{H}$, and $\mathrm{Mu}$ in water has been investigated with high precision measurement of spinexchange dephasing in $\operatorname{EPR}(\mathrm{H}, \mathrm{D})$ and MuSR $(\mathrm{Mu})$ experiments. Modern molecular dynamics simulations of these atoms in water calculate the $g_{\mathrm{HO}}(r)$ radial distribution functions, allowing a choice for the effective reaction distance in the $\mathrm{H}+$ $\mathrm{H}$ recombination reaction. This provides the calibration necessary to deduce the absolute $\mathrm{H}$-atom diffusion coefficient $\left(D_{\mathrm{H}}=1.0 \pm 0.2 \times 10^{-8} \mathrm{~m}^{2} \mathrm{~s}^{-1}\right.$ at $\left.298 \mathrm{~K}\right)$ in light water from reaction rate information. With this calibration, it becomes possible to self-consistently convert the small isotopic variation of spin exchange in $\mathrm{D}, \mathrm{H}, \mathrm{Mu}$ encounters with $\mathrm{Ni}^{2+}$ into a small isotopic variation of the diffusion coefficients in $90 \% \mathrm{D}_{2} \mathrm{O}$ and to compare $\mathrm{H}$-atom diffusion in $90 \% \mathrm{D}_{2} \mathrm{O}$ and $100 \% \mathrm{H}_{2} \mathrm{O}$. 6

The diffusion of these light hydrophobic atoms in water does depend on their mass, although not directly on the square root of mass. The (mass-independent) Stokes-Einstein formula for diffusion of large solute in smaller solvent is clearly inapplicable and irrelevant. The general experimental behavior conforms very well to the RPMD study of Markland et al., ${ }^{10}$ who decompose the overall diffusion into a (mass-independent) "cavity" diffusion component, whereby the light atoms merely follow the diffusion of the water cavity in which they reside, and a (mass-dependent) "hopping" component, in which the light atoms hop between adjacent cavities. The only qualitative discrepancy with the study of Markland et al. is that we find that the $\mathrm{Mu}$ atom diffuses $43 \%$ faster than the $\mathrm{H}$ atom in $90 \%$ $\mathrm{D}_{2} \mathrm{O}$ solvent, where the RPMD simulation with classical TIP4P/2005 water predicts that $\mathrm{Mu}$ should diffuse slower than $\mathrm{H}$ because "quantum swelling" inhibits the hopping mechanism. We have carried out an RPMD simulation of $\mathrm{H}$ and $\mathrm{Mu}$ in a quantized, flexible (anharmonic) water model developed by Habershon et al. ${ }^{39}$ using a spherical Lennard-Jones 14-7 H 6 atom versus water potential derived from $a b$ initio simulations by Pomogaeva and Chipman. ${ }^{9}$ In this fully quantized water 6 simulation, the ratio $D_{\mathrm{Mu}} / D_{\mathrm{H}}$ is 1.8 , similar to the fully classical result of Markland et al. ${ }^{10}$ The muonium diffusion result is apparently much more sensitive to details of the short-range potential than is the $\mathrm{H}$-atom result.

\section{Author information}

\section{Corresponding Author}

*E-mail: bartels.5@nd.edu. Phone: +01 574631 5561. Fax: +01 5746318068.

\section{Notes}

The authors declare no competing financial interest.

\section{Acknowledgments}

Work at Argonne National Laboratory was performed under the auspices of the Office of Science, Division of Chemical Science, US-DOE under contract number W-31-109-ENG-38. Muon work was conducted at and supported by the Paul Scherrer Institute in Villigen, Switzerland. Work at Notre Dame was supported by the Division of Chemical Sciences, Geosciences, and Biosciences, Office of Basic Energy Sciences of the U.S. Department of Energy through award DE-FC0204ER15533. This is manuscript number 5088 of the Notre Dame Radiation Laboratory.

\section{References}

(1) de Grotthuss, C. J. T. Sur la décomposition de l'eau et des corps qu'elle tient en dissolution à l'aide de l'électricité galvanique. Ann. Chim. 1806, LVIII, 54-73.

(2) Marx, D.; Tuckerman, M. E.; Hutter, J.; Parrinello, M. The Nature of the Hydrated Excess Proton in Water. Nature 1999, 397, 601-604.

(3) Benderskii, V. A.; Krivenko, A. G.; Rukin, A. N. Anomalous Mobility of Hydrogen and Deuterium Atoms in Aqueous-Solutions of Electrolytes. High Energy Chem. 1980, 14, 303-308. 
(4) Benderskii, V. A.; Krivenko, A. G. Diffusion of Hydrogen and Deuterium Atoms in Water. Russ. J. Electrochem. 1996, 32, 663-669.

(5) Tyrell, K. R. H.; Harris, K. R. Diffusion in Liquids; Butterworths: London, 1984.

(6) Feinauer, A.; Majer, G.; Seeger, A. The Diffusion of Li-6 and Li7 and the Isotope Effect in Liquid Lithium. J. Phys.: Condens. Matter 1994, 6, L355-L360.

(7) Tse, J. S.; Klein, M. L. Are Hydrogen-Atoms Solvated by Water Molecules? J. Phys. Chem. 1983, 87, 5055-5057.

(8) Deraedt, B.; Sprik, M.; Klein, M. L. Computer-Simulation of Muonium in Water. J. Chem. Phys. 1984, 80, 5719-5724.

(9) Pomogaeva, A.; Chipman, D. M. Hydrogen Atom in Water from Ambient to High Temperatures. J. Phys. Chem. B 2013, 117, 1653016541.

(10) Markland, T. E.; Habershon, S.; Manolopoulos, D. E. Quantum Diffusion of Hydrogen and Muonium Atoms in Liquid Water and Hexagonal Ice. J. Chem. Phys. 2008, 128, 194506.

(11) Gai, H. D.; Garrett, B. C. Path-Integral Calculations of the FreeEnergies of Hydration of Hydrogen Isotopes (H, D, and Mu). J. Phys. Chem. 1994, 98, 9642-9648.

(12) Kirchner, B.; Stubbs, J.; Marx, D. Fast Anomalous Diffusion of Small Hydrophobic Species in Water. Phys. Rev. Lett. 2002, 89, 215901.

(13) Roduner, E.; Percival, P. W.; Han, P.; Bartels, D. M. Isotope and Temperature Effects on the Hyperfine Interaction of AtomicHydrogen in Liquid Water and in Ice. J. Chem. Phys. 1995, 102, 5989-5997.

(14) Roduner, E. Hydrophobic Solvation, Quantum Nature, and Diffusion of Atomic Hydrogen in Liquid Water. Radiat. Phys. Chem. 2005, 72, 201-206.

(15) Ohtaki, H.; Radnai, T. Structure and Dynamics of Hydrated Ions. Chem. Rev. 1993, 93, 1157-1204.

(16) Walker, D. C. Muonium - A Light Isotope of Hydrogen. J. Phys. Chem. 1981, 85, 3960-3971.

(17) Buxton, G. V.; Greenstock, C. L.; Helman, W. P.; Ross, A. B.; Tsang, W. Critical-Review of Rate Constants for Reactions of Hydrated Electrons, Hydrogen-Atoms and Hydroxyl Radicals $\left(\mathrm{OH} / \mathrm{O}^{-}\right)$in Aqueous-Solution. J. Phys. Chem. Ref. Data 1988, 17, 513-886.

(18) Molin, Y. N.; Salikhov, K. M.; Zamaraev, K. I. Spin Exchange. Principles and Applications in Chemistry and Biology; Springer Verlag: New York, 1980.

(19) Ghandi, K.; Addison-Jones, B.; Brodovitch, J. C.; McKenzie, I.; Percival, P. W.; Schuth, J. Near-Diffusion-Controlled Reactions of Muonium in Sub- and Supercritical Water. Phys. Chem. Chem. Phys. 2002, 4, 586-595.

(20) Han, P.; Bartels, D. Encounters of $\mathrm{H}$ and D Atoms with $\mathrm{O}_{2}$ in Water. In Ultrafast Reaction Dynamics and Solvent Effects: Royaumont, France 1993; Gauduel, Y., Rossky, P. J., Eds.; AIP Conference Proceedings 298; American Institute of Physics: New York, pp 72-84.

(21) Sehested, K.; Christensen, H. The Rate Constant of the Biomolecular Reaction of Hydrogen Atoms at Elevated Temperatures. Radiat. Phys. Chem. 1990, 36, 499-500.

(22) Spinks, J. W. T.; Woods, R. J. An Introduction to Radiation Chemistry, 3rd ed.; Wiley Interscience: New York, 1990.

(23) Elliot, A. J.; Bartels, D. M. The Reaction Set, Rate Constants and gValues for the Simulation of the Radiolysis of Light Water over the Range $20^{\circ}$ to $350{ }^{\circ} \mathrm{C}$ Based on Information Available in 2008; Report AECL 153-127160-450-001; Atomic Energy of Canada, Ltd.: Mississauga, Ontario, 2009.

(24) Han, P.; Bartels, D. M. H-Atom Reaction-Rates in Solution Measured by Free Induction Decay Attenuation. Chem. Phys. Lett. 1989, 159, 538-548.

(25) Lossack, A. M.; Roduner, E.; Bartels, D. M. Kinetic Isotope Effects in $\mathrm{H}$ and $\mathrm{D}$ Abstraction Reactions from Alcohols by $\mathrm{D}$ atoms in Aqueous Solution. J. Phys. Chem. A 1998, 102, 7462-7469.

(26) Roduner, E.; Bartels, D. M. Solvent and Isotope Effects on Addition of Atomic-Hydrogen to Benzene in Aqueous-Solution. Ber. Bunsen-Ges. Phys. Chem. Chem. Phys. 1992, 96, 1037-1042.
(27) Mezyk, S. P.; Bartels, D. M. Direct EPR Measurement of Arrhenius Parameters for the Reactions of $\mathrm{H}$. Atoms with $\mathrm{H}_{2} \mathrm{O}_{2}$ and D. Atoms with $\mathrm{D}_{2} \mathrm{O}_{2}$ in Aqueous Solution. J. Chem. Soc., Faraday Trans. 1995, 91, 3127-3132.

(28) Mezyk, S. P.; Bartels, D. M. Rate-Constant and ActivationEnergy Measurement for the Reaction of Atomic-Hydrogen with Methanol, Iodomethane, Iodoethane, and 1-Iodopropane in AqueousSolution. J. Phys. Chem. 1994, 98, 10578-10583.

(29) Freed, J. H.; Pedersen, J. B. The Theory of Chemically Induced Dynamic Spin Polarization. Adv. Magn. Opt. Reson. 1976, 8, 1-84.

(30) Bartels, D. M.; Chiu, T. M.; Trifunac, A. D.; Lawler, R. G. Direct Observation of Initial Polarization (CIDEP) in H-Atoms and D-Atoms Produced in Water Radiolysis. Chem. Phys. Lett. 1986, 123, 497-501.

(31) Bartels, D. M.; Craw, M. T.; Han, P.; Trifunac, A. D. H/D Isotope Effects in Water Radiolysis 0.1. Chemically-Induced Dynamic Electron Polarization in Spurs. J. Phys. Chem. 1989, 93, 2412-2421.

(32) Bartels, D. M.; Trifunac, A. D.; Lawler, R. G. Observations of Heisenberg Spin Exchange between Reactive Free-Radicals. Chem. Phys. Lett. 1988, 152, 109-115.

(33) Bartels, D. M.; Lawler, R. G.; Trifunac, A. D. Electron $T_{1}$ Measurements in Short-Lived Free-Radicals by Dynamic Polarization Recovery. J. Chem. Phys. 1985, 83, 2686-2707.

(34) Han, P.; Bartels, D. M. H/D Isotope Effects in Water Radiolysis 0.3. Atomic-Hydrogen in Acidic $\mathrm{H}_{2} \mathrm{O} / \mathrm{D}_{2} \mathrm{O}$ Mixtures. J. Phys. Chem. 1991, 95, 9370-9374.

(35) Senba, M. Electron Spin Exchange with $\mathrm{O}_{2}$ : Effects on the Muon Spin Rotation, the Electron Spin Resonance, and the Positronium Lifetime. Phys. Rev. A: At., Mol., Opt. Phys. 1995, 52, 4599-4615.

(36) Verma, N. C.; Fessenden, R. W. Time Resolved ESR Spectroscopy 0.4. Detailed Measurement and Analysis of ESR Time Profile. J. Chem. Phys. 1976, 65, 2139-2155.

(37) Roduner, E.; Tregenna-Piggott, P. L. W.; Dilger, H.; Ehrensberger, K.; Senba, M. Effect of Mass on Particle Diffusion in Liquids Studied by Electron-Spin Exchange and Chemical-Reaction of Muonium with Oxygen in Aqueous-Solution. J. Chem. Soc., Faraday Trans. 1995, 91, 1935-1940.

(38) Eisenberg, D.; Kauzmann, W. The Structure and Properties of Water; Oxford University Press: Oxford, U.K., 1969.

(39) Habershon, S.; Markland, T. E.; Manolopoulos, D. E. Competing Quantum Effects in the Dynamics of a Flexible Water Model. J. Chem. Phys. 2009, 131, 024501.

(40) Diffusion-Limited Reactions; Rice, S. A., Ed.; Elsevier: Amsterdam, 1985; Vol. 25, pp 404.

(41) Chipman, D. M. Water from Ambient to Supercritical Conditions with the AMOEBA Model. J. Phys. Chem. B 2013, 117, $5148-5155$.

(42) Ren, P. Y.; Ponder, J. W. Temperature and Pressure Dependence of the AMOEBA Water Model. J. Phys. Chem. B 2004, 108, 13427-13437.

(43) Ren, P. Y.; Ponder, J. W. Polarizable Atomic Multipole Water Model for Molecular Mechanics Simulation. J. Phys. Chem. B 2003, 107, 5933-5947.

(44) Han, P.; Bartels, D. M. Temperature Dependence of Oxygen Diffusion in $\mathrm{H}_{2} \mathrm{O}$ and $\mathrm{D}_{2}$ O. J. Phys. Chem. 1996, 100, 5597-5602.

(45) Friedman, H. L.; Holz, M.; Hertz, H. G. EPR Relaxations of Aqueous $\mathrm{Ni}^{2+}$ Ion. J. Chem. Phys. 1979, 70, 3369-3383.

(46) Manolopoulos, D. E. Personal communication with D. M. Bartels, 2014. 